\title{
Atuação do fisioterapeuta no tratamento da hemorragia peri- intraventricular em recém-nascidos
}

Na evolução da assistência neonatal, ocorreu um grande esforço para a maior sobrevida de recém-nascidos prematuros. Diante desta necessidade da fisioterapia respiratória as unidades de terapia intensiva neonatal (UTIN) se tornaram cada vez mais importantes no que diz respeito aos distúrbios respiratórios, que são responsáveis pela maio as unidades de terapia intensiva neonatal (UTIN) se tornaram cada vez mais importantes no que diz respeito aos distúrbios respiratórios, que são responsáveis pela maior respeito ao tratamento da Hemorragia Perintraventricular (HIPV) em recém-nascidos, sendo levantadas técnicas e tipos de tratamentos desta patologia. Foi realizada uma revisão de literatura, utilizando as bases de dados da BIREME, SCIELO, LILACS E PUBMED, dos quais foram consultados artigos de revistas e jornais no período de 1993 a 2013 , que tratam dos principais conceitos utilizados neste estudo, a fim de embasar teoricamente a pesquisa. Foram utilizados como descritores para a realização da pesquisa nos periódicos, os termos: Fisioterapeuta; Hemorragia Peri-Intraventricular; e Recém-nascidos, sendo a data das pesquisas feitas entre os meses de setembro e outubro do ano de 2017. A fisioterapia respiratória atua tanto na prevenção, quanto no tratamento das doenças respiratórias, sendo utilizado de diversas técnicas e procedimentos terapêuticos ao nível ambulatorial e hospitalar nas Unidades de Terapia Intensiva, com o objetivo de estabelecer/restabelecer o padrão respiratório para reduzir os gastos energéticos no momento da ventilação, possibilitando ao indivíduo realizar diferentes atividades de vida diária sem que tenha grandes transtornos e/ou repercussões negativas em seu sistema respiratório. Diante desta situação a assistência fisioterapêutica feita em recém-nascido tende a apresentar características próprias referentes ao seu peso; à imaturidade e às doenças prevalentes nessa população. Diante dessas particularidades, evidenciam-se dificuldades que exigem cuidados especiais pelo fisioterapeuta junto a recém-nascidos prematuros em unidades de terapia intensiva.

Palavras-chave: Fisioterapeuta; Hemorragia Peri-Intraventricular; Recém-nascidos.

\section{Physiotherapist's performance in the treatment of peri- intraventricular hemorrhage in newborns}

\begin{abstract}
In the evolution of neonatal care, a great effort was made for the greater survival of preterm infants. In view of this need for respiratory physiotherapy, neonatal intensive care units (NICUs) have become increasingly important with regard to respiratory disorders, which are responsible for the greater portion of problems related to prematurity. This article has as main objective to make bibliographical surveys regarding the physiotherapist's performance regarding the treatment of Perintraventricular Hemorrhage (HIPV) in newborns, being raised techniques and types of treatments of this pathology. A review of the literature was carried out, using BIREME, SCIELO, LILACS AND PUBMED databases, from which articles of magazines and newspapers were consulted from 1993 to 2013, which deal with the main concepts used in this study, in order to support theoretically the research. The following terms were used as descriptors for the research in the periodicals: Physiotherapist; Peri-Intraventricular Hemorrhage; and newborns, being the date of the research done between the months of September and October of the year 2017. Respiratory physiotherapy acts both in the prevention and in the treatment of respiratory diseases, being used of various techniques and therapeutic procedures at the outpatient level and in the Intensive Care Units, aiming to establish / restore the respiratory pattern to reduce energy expenditure at the time of ventilation, enabling the individual to perform different activities of daily living without having major disorders and / or negative repercussions on their respiratory system. In view of this situation, physical therapy assistance in newborns tends to present characteristics related to their weight; immaturity and diseases prevalent in this population. In view of these particularities, difficulties that require special care by the physiotherapist with premature newborns in intensive care units are evidenced.
\end{abstract}

Keywords: Physiotherapist; Peri-Intraventricular Hemorrhage; Newborns.

\section{Topic: Fisioterapia}

Reviewed anonymously in the process of blind peer.

Anderson Fazolo Watte (D)

Faculdade Guaraí, Brasil

http://lattes.cnpq.br/5570355394416715

http://orcid.org/0000-0003-1638-1955

andersonwatte@hotmail.com

Rivia da Silva Pereira Miranda (i)

Faculdade Guaraí, Brasil

http://lattes.cnpq.br/5553466513308958

http://orcid.org/0000-0002-3350-2686

riviamiranda@autlook.com

Daniela Maristane Vieira Lopes Maciel (1D

Faculdade Guaraí, Brasil

http://lattes.cnpq.br/0591590391105455

http://orcid.org/0000-0001-5934-0219

danimacielfisio@ibest.com.br

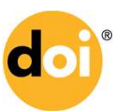

DOI: 10.6008/SPC2236-9600.2017.002.0006
Received: 18/12/2017

Approved: 27/12/2017
Referencing this:

WATTE, A. F.; MIRANDA, R. D. P.; MACIEL, D. M. V. L.. Atuação do fisioterapeuta no tratamento da hemorragia peri-intraventricular em recém-nascidos. Scire Salutis, v.7, n.2, p.50-57, 2017. DOI: http://doi.org/10.6008/SPC2236-9600.2017.001.0006 


\section{INTRODUÇÃO}

A maior sobrevida de recém-nascidos pré-termo determinou, de acordo com o estudo de Luu et al. (2009), maior risco de problemas neurológicos relacionados a hemorragias intracranianas. A hemorragia peri e intraventricular é a afecção neurológica mais importante do período neonatal, acometendo principalmente o recém-nascido (RN) pré-termo (TAVARES, 1998) com peso de nascimento menor que 1500g, ocasionando graves sequelas motoras e intelectuais. É uma das causas mais frequentes de agressão ao sistema nervoso central neste período (LUU et al., 2009).

De acordo com a definição da Organização Mundial da Saúde (OMS), o limite inferior da viabilidade fetal situa-se na $20^{\circ}$ semana (BRASIL, 2011). Desta forma, o parto pré-termo, também denominado parto prematuro, é o parto de um bebê antes das 37 semanas de idade gestacional (SASSÁ, 2014). Um dos marcos da atenção a população de bebês pré-termo, teve seu avanço nas ciências médicas juntamente com a implantação das modernas unidades de terapia intensiva neonatais equipadas com tecnologia de ponta que reduzem a mortalidade destes bebês. Por outro lado, o cuidado presente nestes lugares altamente qualificados e especializados, tem contribuído com o aparecimento de iatrogênicas no processo de crescimento e desenvolvimento destas crianças (RUGOLO, 2005).

A Fisiopatologia da HIPV está intimamente ligada ao evento da prematuridade e a matriz germinativa, que é considerada o principal local de origem do processo hemorrágico. A matriz germinativa dá origem aos neuroblastos ou células germinais do cérebro, entre a $10^{\circ}$ e a $20^{\circ}$ semana de idade gestacional, e aos glioblastos no terceiro trimestre, que em seguida se diferenciam em astrócitos e oligodendrócitos. Ao final do $2^{\circ}$ trimestre da gravidez, a matriz germinativa é uma região com alta atividade metabólica e a parede endotelial nessa área ainda está imatura e frágil. Essas vulnerabilidades anatômicas, associadas à imaturidade de auto regulação vascular nessa época, contribuem para a suscetibilidade da matriz germinativa à hemorragia em resposta a flutuações na pressão cardiovascular (RUGOLO, 2005).

Na evolução da assistência neonatal, ocorreu um grande esforço para a maior sobrevida de recémnascidos prematuros. Diante desta necessidade da fisioterapia respiratória as unidades de terapia intensiva neonatal (UTIN) se tornaram cada vez mais importantes para intervir nos distúrbios respiratórios, que são responsáveis pela maior parcela problemas relacionadas à prematuridade (BALBINO, 2004)

A fisioterapia neonatal acompanha o recém-nascido de alto risco necessitando de um aperfeiçoamento específico para melhor entendimento das peculiaridades neonatais. Realiza a estimulação precoce ajudando na redução do tempo de permanência nos hospitais, diminuindo, assim, as eventuais sequelas, o que contribuirá para o aumento das respostas fisiológicas e metabólicas aos procedimentos aplicados durante o período de internação (PRADO et al., 2012).

A fisioterapia neonatal é uma assistência recente, porém apesar de ter evoluído durante sua utilização com métodos mais eficazes, apresenta divergências no que diz respeito ao uso de recursos terapêuticos e seus possíveis risco benefício nos neonatos prematuros (MICHELIN et al., 2013). Os métodos que verificam os recursos fisioterapêuticos nos recém-nascidos prematuros (RNPT) são escassos, se forem 
levar em consideração os profissionais a muitas vezes realizar erroneamente, recursos que são comprovados na aplicação à população pediátrica e adulta (MICHELIN et al., 2013). Este artigo tem como principal objetivo fazer levantamentos bibliográficos referentes a atuação do fisioterapeuta no tratamento da Hemorragia Perintraventricular em recém-nascidos prematuros.

\section{METODOLOGIA}

Foi realizada uma revisão de literatura, utilizando as bases de dados: da BIREME, SCIELO, LILACS E PUBMED, dos quais foram consultados artigos de revistas e jornais no período de 1993 a 2013 que tratam dos principais conceitos utilizados neste estudo a fim de embasar teoricamente a pesquisa. Os descritores em saúde utilizados foram: Fisioterapeuta, Hemorragia Peri-Intraventricular e Recém-nascidos, sendo a data das pesquisas feitas entre os meses de Setembro e Outubro do ano de 2017.

\section{RESULTADOS E DISCUSSÃO}

\section{A hemorragia peri-intraventricular em recém-nascidos}

A morbidade infantil no Brasil não tem se alterado, mantendo-se constante nos últimos anos mesmo com os avanços tecnológicos na saúde, que tem o importante papel de redução da mortalidade infantil, principalmente nas Unidades de Terapia Intensiva Neonatal (DORLING, 2006). A Hemorragia Periintraventricular, que possui origem mais comum na matriz germinativa e mais raramente no plexo coroide (LOBO et al., 2012), é uma das principais lesões que acometem os recém-nascidos, destacando-se aqueles que nascem com muito baixo peso (ABREU, 2004; ABREU, 1998; ABREU et al., 2006; STOPIGLIA et al., 1999; ABREU et al., 2007), sendo que aproximadamente $50 \%$ dos casos ocorrem nas primeiras $24 \mathrm{~h}$ de vida (BERTAGNI, 2008).

Os RNs pré-termos possuem mecanismos metabólico, químico e neuronal complexo muito deficientes (GIANTONIO et al., 2001), o que pode explicar seu maior risco de HPIV, pois o fluxo sanguíneo cerebral envolve esses mecanismos (LOBO et al., 2012). Segundo Rowland (1999), a HPIV apresenta-se no sangramento da matriz germinativa subependimária estando no tálamo e o caudado, nas proximidades dos forames de Monro. É nesta parte vascularizada por inúmeras arteríolas e veias, que possui vários sistemas de drenagem que aparece uma grande proporção do fluxo sanguíneo cerebral que é direcionado para estas áreas.

De acordo com a ocasião do termo, Rowland (1999) mostra que existe uma remodelação do leito vascular para irrigação do córtex, passando a ser uma área de maior diferenciação nesta época. Desta forma o fenômeno provoca a involução da matriz germinativa. As consequências da hemorragia periintraventricular podem ser muito agressivas, principalmente quando o diagnóstico é feito tardiamente. Algumas de suas consequências são as paralisias cerebrais e o retardo mental. As principais alterações neurológicas são as relacionadas às motoras (AIROLDI et al, 2009). 
Outra complicação seria também a hidrocefalia pós-hemorrágica (MOURA FILHO et al., 2006). Dos recém-nascidos que apresentam hemorragia Peri-intraventricular, mais de $60 \%$ e $30 \%$ apresentam respectivamente dilatação ventricular não progressiva e não progressiva lenta secundária, apresentando coágulos e impedindo a circulação. O prognóstico da hidrocefalia está relacionado com a gravidade da hemorragia (MARGOTTO, 2009). O diagnóstico precoce seria o intermédio pelo qual se podem diminuir às sequelas da morbidade. Além disso, uma das principais medidas a serem tomadas é a prevenção da prematuridade (SILVEIRA et al., 2005), principalmente no que diz respeito à cobertura do atendimento prénatal, a qualidade das consultas e as medidas perinatais em geral.

Para o diagnóstico da HPIV é necessário, além da deteç̧ão de alterações neurológicas, realizar a ultrassom transfontanela (PONTE et al., 2003), este deve ser realizado em todos os recém-nascidos prétermo devido à grande possibilidade de ocorrência nos mesmos (TAVARES et al., 1998). O exame possui baixo custo e há facilidade de transporte do aparelho, o exame pode ser realizado no leito da UTIN, diferentemente de outros exames como a tomografia computadorizada e a ressonância magnética (MOURA FILHO et al., 2006; LOBO et al., 2012). Existem muitos fatores que podem estar associados à hemorragia, tais como: maternos, obstétricos, perinatais, e fatores particulares dos recém-nascidos (LOBO et al., 2012).

Os fatores maternos e obstétricos são os que podem contribuir para um parto precoce, tais como o pré-natal inadequado, hipertensão arterial, diabetes mellitus, gemelaridade. Os perinatais são: parto prolongado, parto vaginal e sofrimento fetal (LOBO et al., 2012). Outros agentes que estão associados a HPIV são idade gestacional (GUZMAN et al., 2010), sexo masculino, a necessidade de VMI, presença de infecção, entre outros (GUZMAN et al., 2010).

Na visualização das diferentes RP, diante de uma manobra, tem levantado alguns questionamentos acerca de uma possível avaliação de escolhas, onde novas técnicas mais criteriosas e individualizadas possam ser criadas (NICOLAU et al, 2007). Desta forma, com a aplicação de boas práticas no que diz respeito ao cuidado ao neonato, ainda há um crescimento da ocorrência de sequelas e disfunções na evolução dos RNs, por que a criança nascida prematura se apresenta susceptível a uma variedade de problemas no seu neurodesenvolvimento, desta forma tem sido descritos como as 'novas morbidades' do RNPT de baixo peso (NICOLAU et al, 2007). Segundo Silveira et al. (2005) os RNPT que sobrevivem ao período neonatal se apresentam com um risco elevado de alterações no que diz respeito ao seu neurodesenvolvimento.

Assim os distúrbios que ajudam essas alterações estão a: a asfixia perinatal, as infecções, o uso de Ventilação Mecânica Invasiva (VMI) e a HPIV. Nestes tipos de lesões cerebrais do RNPT são múltiplas e nestes casos a HPIV permanece de forma a ser classificada como a lesão mais descrita e conhecida (FORMIGA et al., 2010). Durante a Fisioterapia Respiratória, a ventilação mecânica, sendo ela invasiva ou não-invasiva, apresenta-se constituída dentro de alguns procedimentos, conforme afirmam Jerre et al. (2007), como:

A aspiração traqueal, tendo como utilidade a retirada passiva das secreções;

A percussão e a vibração do tórax, buscando a mobilização de secreções;

A drenagem postural, tendo como intuito de drenar as secreções através da ação da gravidade; 
A compressão brusca do tórax, aumentando o fluxo expiratório;

O posicionamento corporal, otimizando a relação ventilação/perfusão, tendo o intuito de aumentar o volume pulmonar e reduzir o trabalho respiratório e cardíaco aumentando o clearance mucociliar;

A expansão/reexpansão da região pulmonar, aumentando a pressão e volume alveolar em áreas colabadas;

A hiperinsuflação de maneira manual, realizando insuflação pulmonar com o auxílio de um ressuscitador manual aumentando o fluxo expiratório; e

A terapia com PEEP, sendo utilizado a técnica de pressão positiva, desta forma, ao final da expiração ou pressão positiva contínua nas vias aéreas promovendo a expansão de unidades alveolares colabadas.

\section{A atuação do fisioterapeuta em patologias respiratórias.}

A fisioterapia respiratória atua tanto quanto na prevenção, no que diz respeito ao tratamento das doenças respiratórias, sendo utilizando de diversas técnicas, quanto nos procedimentos terapêuticos, no que diz respeito ao nível ambulatorial, ao nível hospitalar ou no caso de terapia intensiva, tendo como objetivo de estabelecer/restabelecer o padrão respiratório para reduzir os gastos energéticos no momento da ventilação, possibilitando ao indivíduo realizar diferentes atividades de vida diária sem que tenha grandes transtornos e/ou repercussões negativas em seu sistema respiratório (AZEREDO, 1993).

Para isso acontecer é necessário um melhorar clearance mucociliar, tendo a ventilação e prevenindo ou eliminando o acumulo de secreções nas regiões afetadas, melhorando as trocas gasosas, obtendo manter ou melhorar a mobilidade da região da caixa torácica e da parte abdominal (STOLLER, 2000). As técnicas de fisioterapia que se relacionam com os cuidados respiratórios consistem em técnicas manuais, posturais e cinéticas dos componentes tóraco-abdominais que são aplicados isoladamente ou em associação de outras técnicas, que de forma genérica, tem os seguintes objetivos:

Mobilizar e eliminar as secreções pulmonares; melhorar a ventilação pulmonar; promover a reexpansão pulmonar; melhorar a oxigenação e trocas gasosas; diminuir o trabalho respiratório; diminuir o consumo de oxigênio; aumentar a mobilidade torácica; aumentar a forca muscular respiratória; aumentar a endurance; reeducar a musculatura respiratória; promover a independência respiratória funcional; prevenir complicações e acelerar a recuperação do paciente (AZEREDO, 1993).

Na utilização do suporte ventilatório utilizado em UTI, a Ventilação Mecânica (VM), que se apresenta como um método de suporte ao paciente mecânico (respirador artificial) utilizado no tratamento de pacientes que apresentam insuficiência respiratória aguda/crônica agudizada, ajudando-o na sua ventilação em suas trocas gasosas (SCHETTINO, 2007). Classificada com a seguinte terminologia, VMI e Não Invasiva, desta forma, em ambas as modalidades, a ventilação artificial é feita com a aplicação de pressão positiva nas vias aéreas. (CARVALHO et al., 2000)

Neste tipo de suporte os objetivos são priorizados em manter a troca gasosa do paciente e aliviar o trabalho da musculatura respiratória, ainda buscando reverter ou evitar a fadiga da musculatura respiratória e diminuir o consumo de oxigênio, sendo uma técnica que permite a aplicação de terapias específicas. (CARVALHO, 2007). Nos procedimentos chamados de intervencionistas na fisioterapia respiratória, eles são 
descritos como técnicas de higiene brônquica, técnicas de reexpansão e desinflação pulmonar e incentivadores inspiratórios (COSTA, 2010).

Nas técnicas classificadas de higiene brônquica, que estão listadas como sendo aquelas capazes de mobilizar secreções e prover o seu deslocamento. São as seguintes segundo Stoller (2000), ABREU (1998), e ABREU et al. (2006): vibração torácica; compressão torácica; percussão ou tapotagem; drenagem postural; bag-squeezing; Manobra Zeep; e tosse.

De acordo com Abreu (2003), na vibração torácica os seus movimentos oscilatórios rítmicos e rápidos de pequena amplitude, exercidos de maneira que sobre a parede do tórax a intensidade suficiente, é feita para causar vibração em nível bronquial; uma frequência ideal desejada situa-se entre 3 e $55 \mathrm{~Hz}$ e pode ser aplicada de forma manual ou mecânica. No efeito positivo desta técnica é baseada em sua propriedade tixotrópico do muco, onde se liquefazem quando submetido numa constante agitação (ABREU, 2003).

A compressão torácica e uma técnica que consiste na compressão realizada na parede torácica durante a fase expiratória do ciclo ventilatório de forma relativamente brusca objetivando a formação de fluxo turbulento por aceleração do fluxo expiratório intrapulmonar, objetivando a mobilização de secreções (OBERWALDNER, 2000; ETCHES et al., 1978).

A percussão ou tapotagem se apresenta como um movimento de desobstrução brônquica que tem como objetivo a facilitação do clearance mucociliar. O movimento pode ser considerado como um procedimento muco cinético, pois possibilita a remoção de secreção da arvore brônquica e a paralisa nas regiões periféricas levando-a as centrais, onde será expelida pela tosse ou pela aspiração (ABREU, 1997; ABREU, 2000; DOMINGUES, 1995). A percussão e contraindicada durante a presença da manifestação de enfisema intersticial extenso, hemorragia pulmonar e pneumotórax não drenado. Para Abreu (2003) "a drenagem postural e amplamente empregada na fisioterapia respiratória e seus efeitos são oriundos da ação gravitacional".

A técnica bag-squeezing e um recurso fisioterapêutico sendo utilizado em pacientes que apresentam quadro de hipersecreção pulmonar e tampões mucosos que estão necessitando da utilização de ventilação artificial, através de um aparelho de ventilação mecânica invasiva Abreu (2007). Essa manobra consiste na utilização de uma bolsa máscara de hiperinsuflação pulmonar (ambu) e das técnicas de vibração e compressão torácica. $\mathrm{O}$ uso de manômetro de pressão.

\section{Fisioterapia respiratória sobre a pressão arterial RNPT}

O uso frequente do desenvolvimento da fisioterapia neonatal no Brasil, para Stiller (2000) "vem ocorrendo em centros mais desenvolvidos, tem otimizado a utilização de recursos terapêuticos e de padrões de eficiência no tratamento intensivo, colaborando para a redução do tempo de hospitalização, dos custos e da morbidade dos recém-nascidos em terapia intensiva."

Diante desta situação a assistência fisioterapêutica feita em recém-nascido tende a apresentar características próprias referentes ao seu peso; à imaturidade e às doenças prevalentes nessa população. 
Diante dessas particularidades, evidencia-se dificuldades que exigem cuidados especiais no que diz respeito aos recursos fisioterapêuticos (NORRENBERG, 2000).

Inúmeros fatores intra e extravasculares podem concorrer para o sangramento ou o aumento deste, dentre os quais, as convulsões, os episódios de apneia e hipoxemia, a persistência do canal arterial, a ventilação mecânica e, possivelmente, certos procedimentos, como a aspiração traqueal e a fisioterapia respiratória. (STILER, 2000)

\section{CONCLUSÕES}

Podemos considerar que a HPIV se apresenta como uma afecção que ocorre principalmente nos RN prematuros, onde seus mecanismos associados como LPV, lesão hipóxico isquêmica, que resultam em PC e $\mathrm{HPH}$ definido como o aumento ventricular progressivo, que é secundário a HPIV. Desta maneira, existe a necessidade de orientação de uma equipe multidisciplinar desde o período neonatal. Podendo prevenir a sequência do desenvolvimento motor anormal através da instalação de um programa de reabilitação no sentido de obter o máximo benefício que o acompanhamento multidisciplinar e precocemente estas RNs recebam, os cuidados necessários para que as sequelas sejam diminuídas ou até mesmo evitadas.

Sendo assim uma possibilidade de propiciar não somente uma maior sobrevivência a essas RNs, mas também uma sobrevida, com uma possibilidade de integração à sociedade. A fisioterapia neonatal demonstrou ser um procedimento terapêutico sem repercussões deletérias em relação às variáveis fisiometabólicas para o tratamento da população estudada. Desta forma, torna-se imprescindível que o papel do fisioterapeuta dentro das unidades neonatais seja reformulado, deixando de ser somente o profissional que aplica técnicas para a manutenção da higiene brônquica e passe a ser também o profissional que pesquise e questione o real valor da fisioterapia respiratória.

\section{REFERÊNCIAS}

ABREU L. C.; ANGHEBEN, J. M. M.; BRAZ, P. F.; OLIVEIRA, A. G.; FALCÃO M. C.; SALDIVA, P. H. N.. Effect of the neonatal physiotherapy in the heart rate in preterm infant with respiratory distress syndrome after replacement of exogenous surfactant. Arquivos Médicos do ABC, v.31, n.1, p.5-11, 2006.

ABREU, L. C.. A hemorragia peri-intraventricular do recémnascido pré-termo. Fisio Brasil, v.65, n.1, p.38-42, 2004.

ABREU, L. C.. Efeitos terapêuticos da fisioterapia pulmonar e motora em recém-nascidos pré-termo com hemorragia peri-intraventricular. Dissertação (Mestrado em Reabilitação) - Universidade Federal de São Paulo, São Paulo, 1998.

ABREU, L. C.. Effect of the neonatal physiotherapy in the heart rate in preterm infant with respiratory distress syndrome after replacement of exogenous surfactant. Arquivos Médicos do ABC, v.31, n.1, p.5-11, 2006.

ABREU, L. C.. Fisioterapia no período neonatal. Revista UniABC, p.33-37, 1997; 33-7.
ABREU, L. C.. Hemorragia periventricular-intraventricular do recém-nascido pré-termo como fator predisponente de paralisia cerebral. Revista UniABC, p.18-31, 2000.

ABREU, L. C.. Impacto da fisioterapia neonatal em recémnascidos pré-termo com doença pulmonar das membranas hialinas em ventilação mecânica e pós-reposição de surfactante exógeno. Tese (Doutorado em Fisioterapia) Universidade Federal de São Paulo, São Paulo, 2003.

ABREU, L. C.. Incidência de hemorragia peri-intraventricular em recém-nascidos pré-termo e a relação com o peso ao nascer. Revista Brasileira de Crescimento e Desenvolvimento Humano, v.17, n.2, p.24-30, 2007.

ABREU, L. C.; PEREIRA, V. X.; VALENTI, V. E.; PANZARIN, S. A.; MOURA FILHO, O. F.. Uma visão da prática da fisioterapia respiratória: ausência de evidencia não e evidencia de ausência. Arquivos Médicos do ABC, v.32, n.2, p.76-78, 2007.

AIROLDI, J. M.; SILVA, C. B. M.; SOUZA, T. C. R.. Avaliação de recém-nascidos pré-termo com hemorragia peri- 
intraventricular e/ou leucomalácia peri-intraventricular. Revista de Neurociência, v.17, n.1, p.24-29, 2009.

BALBINO, F. S.. Preocupações dos pais de recém-nascidos prematuros com a proximidade da alta da unidade de terapia intensiva neonatal. Dissertação (Mestrado em Ciências) - Universidade Federal de São Paulo, São Paulo, 2004.

BERTAGNI, C. F.. Atuação da fisioterapia intensiva na hemorragia peri-intraventricular no recém-nascido prétermo com baixo peso. Monografia (Especialização em Fisioterapia Intensiva) - Sociedade Brasileira de Terapia Intensiva, São Paulo, 2008.

BRASIL. Ministério da Saúde. Atenção à saúde do recémnascido: guia para os profissionais de saúde. Brasília: Ministério da Saúde, 2011.

CARVALHO, C. R. R.; TOUFEN JUNIOR, C. F.; AIRES, S. Ventilação Mecânica: princípios, análise gráfica e modalidades ventilatórias. Jornal Brasileiro de Pneumologia, v.33, n.2, p.54-70, 2007.

CARVALHO, W. B. DE. KOPELMAN, GURGUEIRA, G. L. BONASSA J. Liberação de pressão de vias aéreas em pacientes pediátricos submetidos à cirurgia cardíaca. Revista da Associação Médica Brasileira, v.46, n.2, p.166-173, 2000.

COSTA, P. T.. Avaliação do impacto da implantação da assistência de fisioterapia respiratória sobre a morbidade de recém-nascidos prematuros de baixo peso. Dissertação (Mestrado em Medicina) - Faculdade de Medicina da Universidade de São Paulo, São Paulo, 2010.

DOMINGUES, S. S.. Fisioterapia respiratória em recémnascido de alto risco. In: Procedimentos técnicos de enfermagem em UTI neonatal. Arquivos Médicos do ABC, Santo André, v.32, 2007.

DORLING, J. S.; FIELD, D. J.. Follow up of infants following discharge from the neonatal unit: structure and process. Early Human Development, n.6, p.82-151, 2006.

ETCHES, P. C.; SCOTT, B.. Chest physiotherapy in the newborn: effect on secretions removed. Pediatrics, v.62, n.5, p.713-715, 1978.

FORMIGA, C. K. M. R.; CEZAR, M.; LINHARES, M. B. M.. Avaliação longitudinal do desenvolvimento motor e da habilidade de sentar em crianças nascidas prematuras. Revista Fisioterapia e Pesquisa, v.17, n.2, p.102-107, 2010.

GIANTONIO, E.; SCHAEFER, C.; MASTROIACOVO, P. P.. Adverse effects of prenatal methimazole exposure. Teratology, v.64, n.1, p.262-266, 2001.

JERRE, G.. Fisioterapia no paciente sob ventilação mecânica. Jornal Brasileiro de Pneumologia, v.33, n.2, p.142-150, 2007.

LOBO, A. H. G.. Hemorragia peri-intraventricular. In: LOBO, A. H. G.. Atenção à saúde do recém-nascido: Guia para os profissionais de saúde. 2 ed. Brasília: Ministério da Saúde, 2012. p.117-132.
LUU, T. M.; MENT, L. R.; SCHENEIDER, K. C.; KATZ, K. H.; ALLAN, W. C.; VOHR, B. R.. Last in effects of preterm birth and neonatal brain hemorrhage eat 12 years of age. Pediatrics, v.123, p.1037-1044, 2009.

MICHELIN, A. S.; PROTO M. C.; BRITO G. M. G.; ANDRADE F. M. D.; BEZERRA A. L.. Efeitos hemodinâmicos da ventilação não-invasiva com máscara facial em prematuros. Revista Fisioterapia e Pesquisa, v.20, n.4, p. 367-372, 2013.

NICOLAU, C. M.; FALCÃO M. C.. Efeitos da fisioterapia respiratória em recém-nascidos: análise crítica da literatura. Revista Paulista de Pediatria, São Paulo, v.25, n.1, 2007.

NORRENBERG, M.; VINCENT J. L.. A profile of European intensive care unit physiotherapists. Intensive Care Medicine, v.26, 2000

OBERWALDNER, B. Physiotherapy for airway clearance in pediatrics. European Respiratory Journal, v.15, n.1, p.196204, 2000 .

PRADO, C.; VALE, L. A.. Fisioterapia Neonatal e Pediátrica. Barueri: Manole, 2012.

ROWLAND, L. P.. Merritt: tratado de neurologia. 9 ed. Rio de Janeiro: Guanabara Koogan, 1997.

RUGOLO, L. M. S. S.. Crescimento e desenvolvimento em longo prazo do prematuro extremo. Jornal de Pediatria, São Paulo, 2005.

SASSÁ, A. H.. Bebês pré-termo: aleitamento materno e evolução ponderal. Revista Brasileira de Enfermagem, v.67, n.4, 2014.

SCHETTINO, G. P. P.. Ventilação mecânica não invasiva com pressão positiva. Jornal Brasileiro de Pneumologia, v.33, n.2, p. 92-105, 2007

SILVA, J. R. O. C.. Aspectos básicos da lesão de isquemia e reperfusão e do pré-condicionamento isquêmico. Acta Cirúrgica Brasileira, v.17, n.3, p.96-100, 2002.

SILVEIRA, R. C.; PROCIANOY, R. S.. Lesões isquêmicas cerebrais no recém-nascido pré-termo de muito baixo peso. Jornal de Pediatria, Porto Alegre, 2005.

STILLER, K.. Physiotherapy in intensive care: towards an evidence-based practice. Chest, Boston, v.118, p.1801-1813, 2000

STOLLER, S. W.. Fundamentos da Terapia Respiratória de Egan. São Paulo: Editora Manole, 2000.

STOPIGLIA, M. S.. Neurological evaluation of neonates with intraventricular and periventricular hemorrhage. Neuropsychiatry Files, v.57, n.2, p.366-370, 1999.

TAVARES, E. C.; CORRÊA F. F.; VIANA M. B.. Fatores de risco para hemorragias peri-intraventriculares em recém-nascidos com peso menor de 2000 gramas. Jornal de Pediatria, Belo Horizonte, v.74, n.1, p.17-24, 1998. 